\section{MS23-04 X-ray nano-diffraction of semiconductor nano-structures for photonic and electronic applications}

\author{
Vincent Favre-Nicolin ${ }^{1,2}$, ELZO AIZARNA Marta ${ }^{1,2,3}$, \\ MANDULA Ondrej 1,2,3,4, MASTROPIETRO Francesca ${ }^{2,3,5}$, \\ CARBONE Gerardina ${ }^{3,6}$, ANDRIEU François ${ }^{7}$, CLAUDON \\ Julien $^{1,2}$, GERARD Jean-Michel ${ }^{1,2}$
}

1. Univ. Grenoble Alpes, France

2. CEA, INAC-SP2M, Grenoble, France

3. ESRF, The European Synchrotron

4. Fondation Nanosciences, Grenoble, France

5. Université Aix-Marseille, Institut Fresnel, France

6. Max IV Laboratory, Lund University, Sweden

7. CEA-LETI, Minatec, Grenoble, France

email: Vincent.Favre-Nicolin@ujf-grenoble.fr

The last 20 years have seen a massive development of crystalline structures with sub-micrometer sizes, either with a simple miniaturization goal, or in order to exploit quantum confinement effects. The study of these objects is a challenge for crystallographers, as their size implies a weak, dif- fuse scattering rather than sharp Bragg peaks. Moreover, nano-structures, either due to the synthesis method or by design, are often heterogeneous and therefore present inhomogeneous strain and com- position 3D fields.

Thanks to the development of focused X-ray optics, it is now possible to measure the scattering from single nano-objects using X-ray Coherent Diffraction Imaging (XCDI) $[1,2]$ and Ptychography. When used in the Bragg geometry, it allows not only to recover the shape (electronic density), but also the deformation field relatively to a perfect lattice, in the case of an inhomogeneous strain. We will illustrate the use of X-ray nanobeams on single homogeneous and heterogeneous nano- structures $[3,4,5]$ used for photonic (single photon emission) and electronic (strained silicon-on- insulator) applications, and discuss the current performance, limits and prospects of the method.

[1] Miao J, Charalambous P, Kirz J \& Sayre D (1999). Nature 400, 342

[2] Newton MC, Leake SC, Harder R \& Robinson IK (2010) Nat. Mater 9, 120

[3] Favre-Nicolin V, Eymery J, Koester R \& Gentile P (2009). Phys Rev B79, 195401.

[4] Favre-Nicolin V et al. (2010) New J. Phys. 12, 35013.

[5] Mastropietro F, Eymery J, Carbone G, Baudot S, Andrieu F \& Favre-Nicolin V (2013) Phys. Rev. Lett. 111,215502

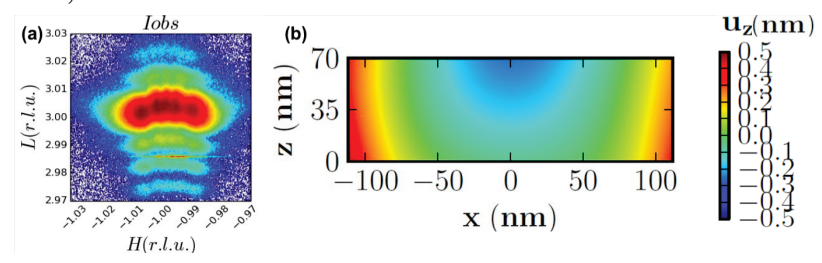

Figure 1. (a) Coherent diffraction pattern from a single strained Silicon-On-Insulator (sSOI) line, with a $225 \times 70 \quad \mathrm{~nm} 2$ cross-section. The curved shape is due to the bending of the strained line. (b) the displacement field refined for this line using the diffraction pattern.[5]

Keywords: Nanostructures, coherent diffraction, semiconductor
MS23-O5 Solvent-nanoparticle interfaces source of solvent restructuring in solvation shells and disorder in nanoparticles

Mirijam Zobel $^{1}$, Simon A.J. Kimber ${ }^{2}$, Reinhard B. Neder ${ }^{1}$

1. Friedrich-Alexander-University Erlangen-Nürnberg, Erlangen, Germany

2. European Synchrotron Radiation Facility (ESRF), Grenoble, France

email: mirijam.zobel@fau.de

Pair distribution function (PDF) measurements have originally been used to study the structure of liquids and glasses. [1] Almost forgotten since, the method experienced a revival with increasing interest in nanoparticles and in situ studies of chemical reactions, where common x-ray diffraction fails. [2] To our knowledge, all previous in-situ PDF studies were undertaken in either aqueous or supercritical solvents [3], although manifold nanoparticle syntheses use organic solvents. The drawback to study nucleation in organic solvents are the direct implications of using them. Not only do they scatter x-rays stronger than water, but more importantly organic molecules possess several internal interatomic distances as well as feature pronounced intermolecular ordering within the bulk solvent. [1] In highly diluted systems as often found in nanoparticle nucleation, these intra- and intermolecular distances of the solvent molecules contribute to more than $99 \%$ of the overall signal in the experimental PDFs. The extraction of the nanoparticle signal hence requires understanding of the organic solvent. It has been theoretically predicted, that solvent molecules restructure at nanoparticle surfaces inside a nanoscopic solvation shell. [4] Within a well-chosen matrix of dispersed nanoparticles ( $\mathrm{ZnO}$, $\mathrm{TiO}_{2}, \mathrm{ZrO}_{2}, \mathrm{Ag}$ ) in the primary alcohols methanol to 1-propanol ${ }^{2}$ as well as in nonpolar hexane, we could for the first time experimentally proof this restructuring effect at solvent-nanoparticle interfaces. The rearrangement of molecules reaches out as far as $2 \mathrm{~nm}$ into the bulk liquid and decays exponentially, see Fig. 1. [5] The interaction of the solvent molecules with the nanoparticle surface does, however, also influence the internal nanoparticle structure during nucleation. In reactions with high supersaturation, surface-bond ligand molecules trap the disorder within the particles before crystalline order is established. We show how different organic ligand molecules influence the crystallization process and that obviously gradual dynamic exchange processes of ligand and solvent molecules at the nanoparticle surface enable relaxation into a crystalline particle.

References:

[1] Zachariasen, W. H., J. Chem. Phys. 3 (1935), 158

[2] Borkiewicz, O. J., et al, Phys. Chem. Chem. Phys. 15 (2013) 8466

[3] Jensen, K. M. O., et al, J. Am. Chem. Soc. 134 (2012), 6785

[4] Spagnoli, D., et al, Geochim. Cosmochim. Acta 73 (2009), 4023

[5] Zobel, M., et al, Science 347 (2015), 292 